\title{
Cell cycle progression is regulated by intertwined redox oscillators
}

Jorgelindo da Veiga Moreira', Sabine Peres², Jean-Marc Steyaert ${ }^{1}$, Erwan Bigan ${ }^{1}$, Loïc Paulevé ${ }^{1,2}$, Marcel Levy Nogueira ${ }^{1,3}$ and Laurent Schwartz ${ }^{1 *}$

\author{
* Correspondence: \\ laurent.schwartz@polytechnique.edu \\ ${ }^{1}$ Ecole Polytechnique, LIX-UMR \\ 7161, Palaiseau, France \\ Full list of author information is \\ available at the end of the article
}

\begin{abstract}
The different phases of the eukaryotic cell cycle are exceptionally well-preserved phenomena. DNA decompaction, RNA and protein synthesis (in late $G_{1}$ phase) followed by DNA replication (in $S$ phase) and lipid synthesis (in $G_{2}$ phase) occur after resting cells (in $G_{0}$ ) are committed to proliferate. The $G_{1}$ phase of the cell cycle is characterized by an increase in the glycolytic metabolism, sustained by high $N \mathrm{ND}^{+} / \mathrm{NADH}$ ratio. A transient cytosolic acidification occurs, probably due to lactic acid synthesis or ATP hydrolysis, followed by cytosolic alkalinization. A hyperpolarized transmembrane potential is also observed, as result of sodium/potassium pump (NaK-ATPase) activity. During progression of the cell cycle, the Pentose Phosphate Pathway (PPP) is activated by increased $\mathrm{NADP}^{+} / \mathrm{NADPH}$ ratio, converting glucose 6-phosphate to nucleotide precursors. Then, nucleic acid synthesis and DNA replication occur in $\mathrm{S}$ phase. Along with $S$ phase, unpublished results show a cytosolic acidification, probably the result of glutaminolysis occurring during this phase. In $G_{2}$ phase there is a decrease in NADPH concentration (used for membrane lipid synthesis) and a cytoplasmic alkalinization occurs. Mitochondria hyperfusion matches the cytosolic acidification at late $G_{1} / S$ transition and then triggers ATP synthesis by oxidative phosphorylation. We hypothesize here that the cytosolic $\mathrm{pH}$ may coordinate mitochondrial activity and thus the different redox cycles, which in turn control the cell metabolism.
\end{abstract}

Keywords: Cell cycle, CCM, REDOX, Intracellular pH, ATP/ADP, NAD(P) ${ }^{+} / \mathrm{NAD}(\mathrm{P}) \mathrm{H}$, HATS, HDACS

\section{Background}

For several years now, a number of studies have been conducted, in the field of the bioenergetic origin of life [1]. Bioenergetics consists in converting nutrients such as carbohydrates, lipids, and proteins, into intermediate metabolites as well as energy for cell survival and finally into de novo building blocks such as nucleic acids, proteins and lipids for cell proliferation. More broadly, cell metabolism is the sum of all the chemical reactions and dynamic exchanges between a cell and its microenvironment. Eukaryotic cells, at least, exhibit two opposite metabolisms: anabolic reactions, which consist in biomass synthesis and catabolic reactions, leading to the breakdown of macromolecules for energetic use. These two aspects of cell metabolism are managed by biochemical and biophysical oscillators, including reductive and oxidative (redox) couples, the most important ones being Nicotinamide Adenine Dinucleotide $\left(\mathrm{NAD}^{+} / \mathrm{NADH}\right)$ and Nicotinamide

(c) 2015 da Veiga Moreira et al. This is an Open Access article distributed under the terms of the Creative Commons Attribution License (http://creativecommons.org/licenses/by/4.0), which permits unrestricted use, distribution, and reproduction in any medium provided the original work is properly credited. The Creative Commons Public Domain Dedication waiver (http:// creativecommons.org/publicdomain/zero/1.0/) applies to the data made available in this article, unless otherwise stated. 
Adenine Dinucleotide Phosphate (NADP $\left.{ }^{+} / \mathrm{NADPH}\right)$, the universal energy carrier, Adenine Triphosphate (ATP/ADP), the transmembrane potential $(\mathrm{Vm})$ and, last but not least, the intracellular $\mathrm{pH}(\mathrm{pHi})$ of the cell. The dynamics of these internal biological rhythms are shown to exhibit oscillatory phenotypes in dividing cells [2].

The intriguing metabolic feature of proliferating cells compared with quiescent ones highlights the well-conserved sequential events characterizing the eukaryotic cell cycle. From the point of view of the central carbon metabolism (CCM), (Fig. 1), the quiescent cells (in $G_{0}$ ) have a basal oxidative metabolism, whereas, in proliferating cells, the carbon flux is rewired to biomass synthesis and cell growth [3]. The latter is enhanced by a high glycolytic rate consuming $\mathrm{NAD}^{+}$and $\mathrm{ADP}$ species for cytoplasmic glucose conversion into pyruvate, generating NADH and ATP molecules. NADH is oxidized back to $\mathrm{NAD}^{+}$through pyruvate conversion into lactate, termed as the Warburg effect after the German Nobel laureate Otto Warburg, and ATP is used as an energy supplier for RNA and protein synthesis in $G_{1}$ of the cell cycle. Glycolysis is then shunted to the pentose phosphate pathway (PPP), generating nucleic acid precursors for DNA replication in the $\mathrm{S}$ phase and NADPH reductive species used later on in the cell cycle progression for membrane lipid synthesis in $G_{2}$. The $G_{2}$ phase is also characterized by full mitochondrial activity, where the citric acid cycle takes place, enabling glucose oxidation and ATP synthesis.

Moreover, current hypothesis support the proton gradient-dependent ATP hydrolysis and synthesis into the cytosol and mitochondria, respectively, as critical events in both transmembrane potential and intracellular $\mathrm{pH}$ oscillation during cell cycle. In this study we aim at juxtaposing interesting results confirming the hypothesis of the pivotal role of pHi on mitochondrial activity and the resulting redox oscillations timing the progression of the cell cycle. For that, we first report the redox phenomena involved in central carbon metabolism and how it regulates the metabolic transitions during the cell cycle progression. Secondly, based on literature reports, we highlight intracellular $\mathrm{pH}$ role in cell metabolism and its potential involvement in "clocking" transitions during the cell cycle.

\section{Cellular redox transitions in CCM during cell cycle progression 1. The metabolic status of quiescent cells in $G_{0}$}

Quiescent cells have a basal oxidative metabolism [3]. They use nutrients such as glucose, protein and fatty acids as main energy supplier to support primary reactions such as amino acids and nucleotide synthesis (see Fig. 1-a) [4]. For that, the glycolytic pathway converts glucose to pyruvate and produces the universal energy transporter in living systems, adenosine triphosphate (ATP). Pyruvate can either be converted into lactate, in the cytosol, or join the tricarboxylic acid (TCA) cycle, taking place in mitochondria. There, ATP is synthesized by oxidative phosphorylation. On the other hand, macromolecules such as proteins and fatty acids are also degraded and join mitochondria for full conversion to ATP, carbon dioxide $\left(\mathrm{CO}_{2}\right)$ and water [3]. The electrochemical energies released during these catabolic reactions are captured by electron carrier species such as nicotinamide adenine nucleotide $\left(\mathrm{NAD}^{+}\right)$, converting it to its respective reduced partner $\left(\mathrm{NADH}, \mathrm{H}^{+}\right)$. $\mathrm{NADH}, \mathrm{H}^{+}$is the electron donor of the mitochondrial electron transport chain (ETC.) during oxidative phosphorylation. It is not clear if NADH 


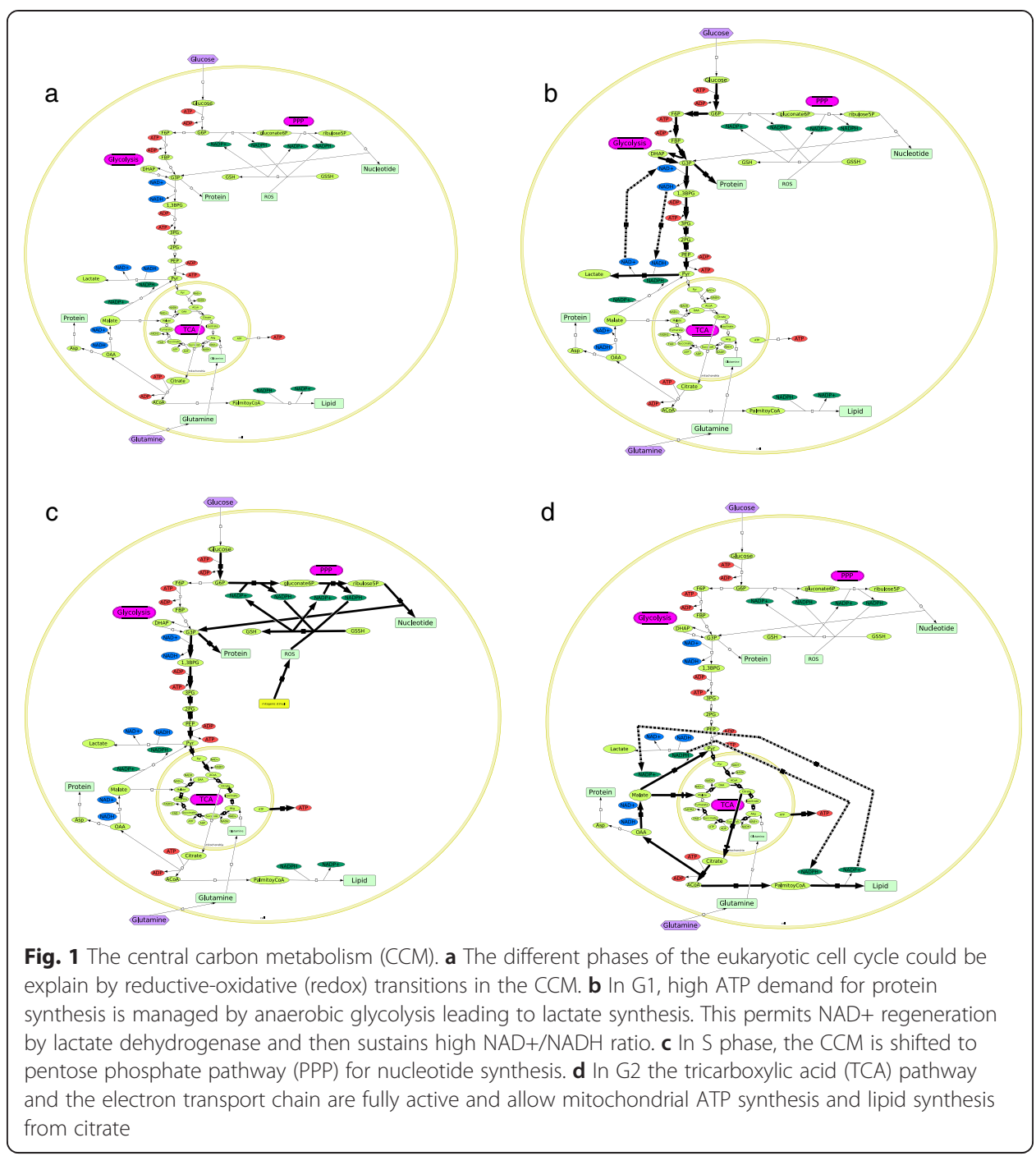

is able to cross the mitochondrial membrane. However, it is reported that specific shuttles such as the malate/citrate shuttle allows mitochondrial NADH regeneration through the TCA cycle [5] (see Fig. 1-a). This basal metabolism allows homeostatic control of high $\mathrm{NAD}^{+} / \mathrm{NADH}$ and low NADP $+\mathrm{NADPH}$ redox ratios [6] in resting cells. On the opposite, in proliferating cells, the basal catabolic metabolism is shifted to anabolism and exhibit oscillatory conversion of these redox species for biomass synthesis and cell growth [3] (see Fig. 2).

\section{Redox oscillation in dividing cells}

2.1. Cell cycle entry into $G 1$ is regulated by the Warburg effect Glycolysis is one of the fundamental pathways of living organisms. It allows the conversion of one molecule of glucose into two pyruvates. During this process, universal energy transporters, such as ATP and NADH, are produced from the oxidative conversion of ADP and NAD ${ }^{+}$, respectively. This pathway is characterized by two phases: the so-called "investment" phase, since it consumes two ATP molecules, and the "payoff" phase, which produces four ATP. The first phase consists in catabolizing one molecule of glucose into two 


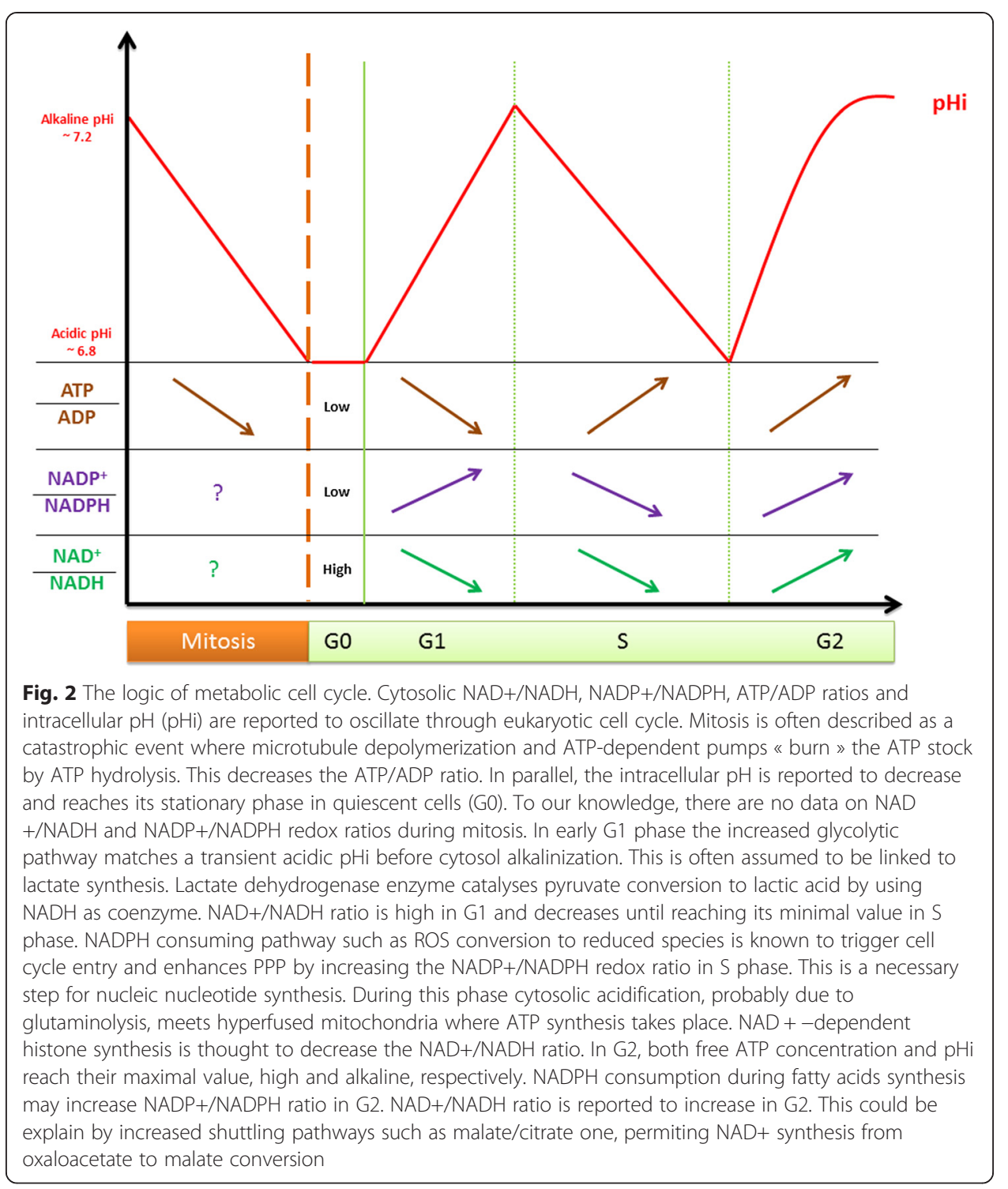

carbon phosphate derivatives, glyceraldehyde 3-phosphate (G3P). The second one gives two pyruvates that will later play a pivotal role in mitochondria-dependent energy production. In one study, Diaz-Moralli and colleagues (2012) assume that the glycolytic pathway mainly occurs during the first growth phase of the eukaryotic cell cycle $\left(G_{1}\right)$ [7]. Indeed, in $G_{1}$ cells grow and synthesize messenger RNA (mRNA) for protein synthesis. Moreover, these studies [8-10] reported the aerobic glycolysis to follow biochemical principles based on "thermodynamic favorability, availability of enzymatic mechanisms and the physicochemical properties of pathway intermediates", meeting the cells' energy demands for cell proliferation.

High glycolytic flux in central carbon metabolism (CCM) occurs when quiescent cells are committed to proliferation [3]. High ATP demand for protein synthesis meets high $\mathrm{NAD}^{+} / \mathrm{NADH}$ redox ratio, allowing glycolysis to persist through the fermentative pyruvate to lactate conversion by lactate dehydrogenase enzyme (Fig. 1-b). This 
consists in a metabolic switch from oxidative phosphorylation, in quiescent cells, to glycolytic phosphorylation, in non-transformed proliferating cells, entering cell cycle $[11,12]$. Calderon-Montano and colleagues [11] highlighted the intracellular $\mathrm{pH}(\mathrm{pHi})$ role in regulating glycolytic genes such as phosphofructokinase-1 (PFK-1). Increased pHi such as observed in cancer cells increases biomass (DNA and protein) synthesis. This anabolic metabolism observed in rapidly-proliferating cells results in redox oscillations of cytoplasmic free ATP concentration, as well as the $\mathrm{NAD}^{+} / \mathrm{NADH}$ ratio $[13,14]$ (Fig. 2). It sustains the aerobic glycolysis observed in G1, just as described by Otto Heinrich Warburg in its seminal work on the glycolytic mode of cancer metabolism [15]. Interestingly, recent studies provide explanations of the Warburg effect, from quantitative models of the metabolic shift in cancer cells, which has the same metabolic signature as normalproliferating cells in G1 phase [16-18]. These are based on analytic rules deciphering the dualistic aspect of proliferating cells metabolism where the abundant resource triggering cell cycle entry favors the glycolytic phenotype, referred as Warburg effect in cancerous tissue. In short, the Warburg effect drives the volumetric growth in G1 by "metabolosomic" biomass synthesis whereas mitochondria activity triggers the surfacic growth.

It is noteworthy that this biphasic growth taking place in proliferating cells is under the control of growth stimuli and redox species oscillation $[19,20]$. In mammalian cells, growth factors and their respective receptors are reported to generate ROS and trigger cell cycle entry $[7,21,22]$. Therefore, moderate ROS formation in late $G_{1}$ is essential for gene transcription and protein synthesis, by modulating DNA accessibility [7]. In fact, an extensive number of studies support that histone acetylation is a pivotal epigenetic program controlling eukaryotic gene transcription [23-25]. In short, transcriptionally active genes are shown to meet hyperacetylated histones, while the "hypoacetylated histone is associated with transcriptionally repressed genes" [25, 26]. Firstly, it is shown that the cytoplasmic $\mathrm{NAD}^{+} / \mathrm{NADH}$ redox ratio optimizes the glycolytic flux (Warburg effect) to ATP and amino acid synthesis. Secondly ATP is used as a supplier of energy for gene transcription and protein synthesis, using these amino acids as building blocks.

2.2. $\mathrm{NADP}^{+} / \mathrm{NADPH}$ ratio regulates the pentose phosphate pathway In normal proliferating cells, one of the first pathways switched on after aerobic glycolysis, is the pentose phosphate pathway (PPP) (Fig. 1-c). This is the main circuit for DNA precursor synthesis and nicotinamide adenine dinucleotide phosphate (NADPH) regeneration, a coenzyme used by the cell for lipid synthesis, in $G_{2}$, and plays the role of reactive oxygen species (ROS) scavenger in $\mathrm{G}_{1} / \mathrm{S}$ transition phase [7, 27-29]. The PPP is characterized by two branches: the oxidative branch, where $\mathrm{NADP}^{+}$is reduced to NADPH by the shunt of glycolysis from glucose 6-phosphate (G6P) conversion into ribulose 5phosphate (R5P) [30], and the non-oxidative branch rewiring the PPP to aerobic glycolysis up to fructose 6-phosphate (F6P) and glyceraldehyde 3-phosphate (G3P).

Interestingly, the PPP has been shown to be regulated by the $\mathrm{NADP}^{+} / \mathrm{NADPH}$ ratio $[27,31,32]$. Therefore, glucose 6-phosphate dehydrogenase (G6PDH) enzyme, catalyzing the conversion of G6P into 6-phosphogluconolactone, has been shown to have an allosteric activity, modulated by the cytoplasmic $\mathrm{NADP}^{+} / \mathrm{NADPH}$ ratio [31]. This is in agreement with these studies [27, 32], which assume that the 
G6PDH catalytic activity is accelerated by an increased NADP ${ }^{+} / \mathrm{NADPH}$ ratio, a result of cellular NADPH consumption by reactive oxygen species (ROS) in S phase.

In this study [29] on changes in the activity of the PPP, the authors showed that NADPH-consuming pathways enhance the pentose phosphate cycle (Fig. 2-c). At least two significant pathways have been identified as major NADPH consuming systems: the glutathione redox cycle (GSH/GSSH) and the lipid synthesis pathway $[6,33,34]$. The reduced glutathione (GSH) is a protective and antioxidant agent that reduce reactive oxygen species (ROS), used in signaling cascades to trigger cell cycle entry (see above) [7], and generates the oxidative species (GSSH). NADPH reduces GSSH and, as a result, they both are converted back to their respective oxidative "partner", GSH and $\mathrm{NADP}^{+}$. In doing so, the glutathione redox cycle enhances the primary pathway using $\mathrm{NADP}^{+}$as a coenzyme: the PPP oxidative branch. The second NADPH-consuming pathway is lipid synthesis, which builds fatty acid blocks from acetyl-CoA.

\section{Mitochondria and their pivotal role in cell anabolic demand in $G_{2}$}

Mitochondrial activity is a key in cell metabolism decision-making and cell cycle progression. As it has been extensively reviewed in this study [35], mitochondria are organelles that have been the subject of many controversies. They were first considered as just a "powerhouse" of the eukaryotic cell, before the pioneering studies deciphering their key role in processes such as development, survival, division, and cell death. Regarding cell division, investigations support and highlight the idea of intertwined relationships between machineries governing mitochondrial dynamics and cell cycle metabolism [36, 37]. It has then been demonstrated that energy transitions occurring in the cell cycle are intrinsically linked to mitochondria sensing parameters, such as the intracellular $\mathrm{pH}$ (pHi) [38] and ATP/ADP ratio [39-41].

Recent studies highlight the mitochondrial morphogenesis at the $G_{1} / S$ transition of the cell cycle. It is also shown that at the $\mathrm{G}_{1} / \mathrm{S}$ checkpoint, mitochondria form a single giant factory for ATP synthesis $[5,42]$. "This energetic boost" is thought to be necessary to increase cyclin $E$ expression in order for the cell to pass the $G_{1} / S$ checkpoint [41]. As stated above, this event could also be interpreted as a necessary step in cell energy supply for protein, nucleic acid, and membrane lipid synthesis (Fig. 1-d). Interestingly enough, these bodies of works support the idea of cell cycle progression meeting the tricarboxylic acid (TCA) cycle and oxidative phosphorylation in $S / G_{2}$ phase transition [7]. The TCA or Krebs cycle, which takes place in mitochondria, in conjunction with oxidative phosphorylation, does indeed allow for carbohydrate oxidation to $\mathrm{CO}_{2}, \mathrm{H}_{2} \mathrm{O}$ and TCA intermediate species. This is also the most efficient route for ATP and lipid precursors' synthesis in the mitochondrial matrix.

The pyruvate accumulated in the cytosol from glycolysis passes through the recently identified specific mitochondrial pyruvate carrier (MPC) and is converted into acetylCoA [43-45]. This reaction is catalyzed by pyruvate dehydrogenase with $\mathrm{NAD}^{+}$as coenzyme. The first step in the TCA cycle is acetyl-CoA conversion into citrate through dehydration. According to the metabolic state of the cell, citrate can shunt to lipid synthesis or continue the Krebs cycle (Fig. 1-d). For that, key enzymes are regulated by negative feedback loops [46]. This includes citrate synthase, isocitrate dehydrogenase and $\alpha$-ketoglutarate dehydrogenase. These enzymes are down-regulated by NADH and 
ATP. Also, this half part of the TCA cycle is the main route for glutaminolysis. This consists of a series of biochemical reactions by which the glutamine amino acid is lysed into glutamate and then $\alpha$-ketoglutarate. From there, there are two possibilities: the oxidative route enables the full Krebs cycle and conversion of $\alpha$-ketoglutarate into succinyl-CoA [47]. The reductive route is the $\alpha$-ketoglutarate conversion to isocitrate and then citrate, the precursor of lipid synthesis. It is interesting to note the reported negative regulation of citrate synthase by succinyl-CoA [46]. Also noteworthy here, is that reductive versus oxidative metabolism mutually exclude each other [48]. At the same time, ATP synthesis through the oxidative phosphorylation chain is required for membrane lipid synthesis in the second growth phase $\left(G_{2}\right)$ (see Fig. 1-d) [7].

Oxidative phosphorylation mainly occurs in the internal membrane of the mitochondria in eukaryotic cells. It uses TCA cycle precursors and co-enzymes, such as $\mathrm{NADH}$ and $\mathrm{FADH}_{2}$, as electron donors for respiration. Through a cascade of reactions, the ETC. complexes trigger the pumping of protons out of the matrix and enable a $\mathrm{pH}$ gradient $(\Delta \mathrm{pH})$ required for ATP synthesis (Fig. 3) [49]. In this redox chain, molecular oxygen $\left(\mathrm{O}_{2}\right)$ is used as the ultimate electron acceptor and ATP is generated as protons moves down its concentration gradient through a well evolutionary-conserved enzyme called ATP synthase, in the inner membrane of mitochondria (reviewed here [49]. This chemiosmotic theory, developed by P. Mitchell [50], explains how NADH and $\mathrm{FADH}_{2}$ oxidation are coupled to ADP phosphorylation into ATP. This is a coupling between oxidation and phosphorylation by a proton gradient across the inner mitochondrial membrane. The oxidative energy from NADH is converted into osmotic energy by proton gradient formation across the membrane, where the intermembrane space is more acidic and the matrix is alkaline. The $\Delta \mathrm{pH}$, which is the $\mathrm{pH}$ difference between the matrix and the intermembrane space, is generated by enzymatic complexes of the ETC. Five complexes have been identified. Only complexes I, III, IV are proton extruders. Four protons are extruded by complexes I and IV and two protons for complex II. The last complex is the ATP synthase catalyzing ADP phosphorylation into ATP against three protons diffusing back to the mitochondrial matrix.

As stated above, the mitochondrial respiratory chain is mainly fueled by the $\mathrm{NAD}^{+} / \mathrm{NADH}$ redox couple. In order for the proliferative cell to maintain high ATP synthesis for cytoskeletal dynamics and membrane lipid synthesis, a symbiotic structure is formed between cytosol and the mitochondria. These are the metabolic shuttles, where one of the most important ones being the malate/citrate shuttle (Fig. 1-d). In fact, while mitochondrial ATP synthesis is high in $G_{2}$, citrate is shuttled out from the Krebs cycle [7, 13]. In the cytosol it is converted into acetyl-CoA and oxaloacetate [3,51]. As mentioned in the sections above, acetyl$\mathrm{CoA}$ is used as a fatty acid precursor for membrane synthesis and cell growth in $\mathrm{G}_{2}$. This well-described pathway consumes NADPH, generated from the malic enzyme and the pentose phosphate pathway [6]. On the other hand, oxaloacetate is converted into malate by consuming NADH and produces $\mathrm{NAD}^{+}$. The reverse reaction occurs in mitochondria, where malate, shuttled-in from cytosol, is converted back to oxaloacetate during the Krebs cycle and generates NADH, the first electron donor of ETC. This forms a full cycle in $\mathrm{G}_{2}$ (Fig. 1-d), where $\mathrm{NAD}^{+} / \mathrm{NADH}$ and $\mathrm{NADP}^{+} / \mathrm{NADPH}$ redox ratios both increase in order to fulfill lipogenesis. 


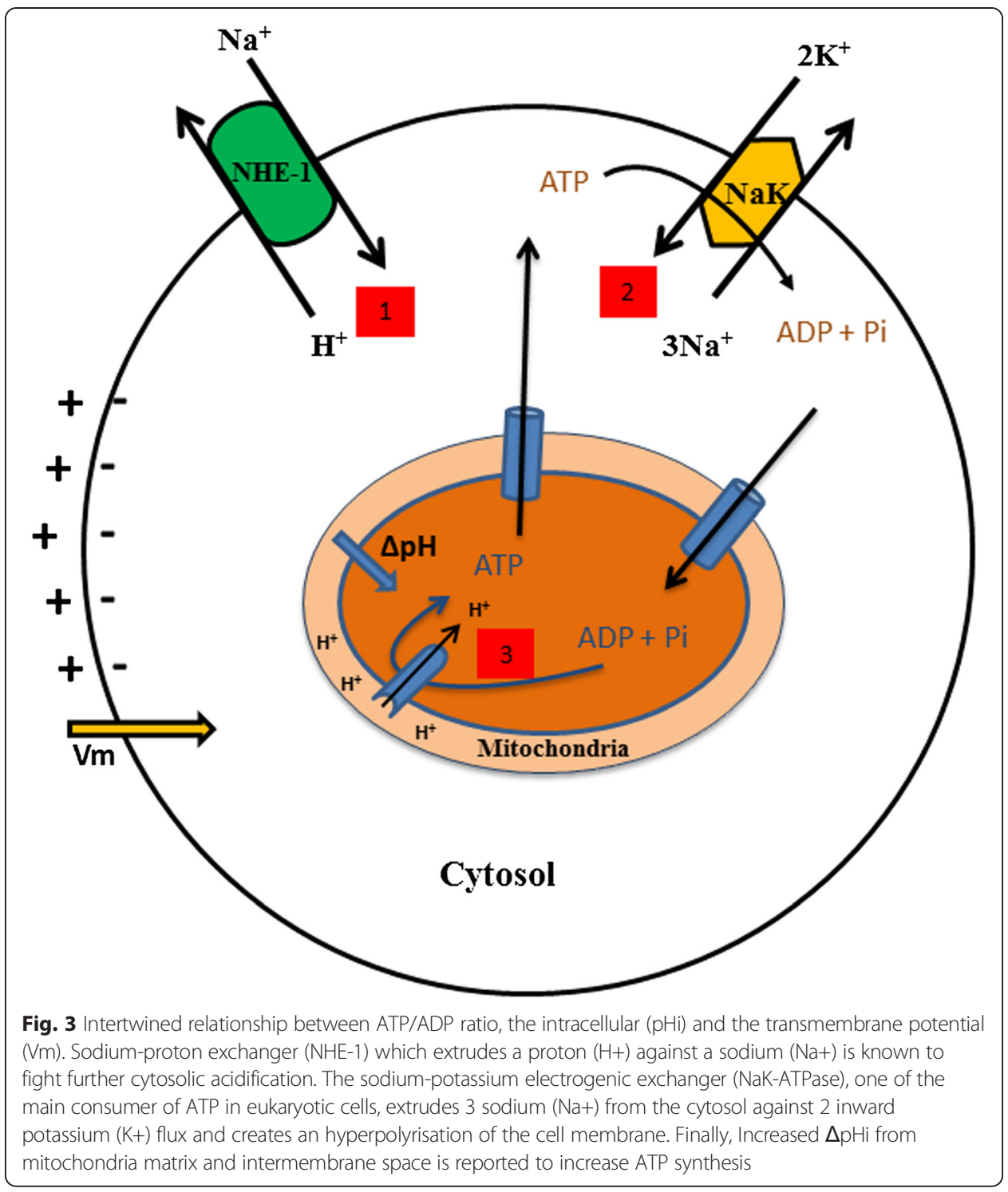

The last but not least cycling parameter of the metabolic cell cycle is intracellular $\mathrm{pH}$, which has been reported to match mitochondrial activity and described as a potential "internal clock" for cell mitosis [52].

\section{Intracellular $\mathrm{pH}$ and ATP/ADP ratio time the metabolic cell cycle}

\section{ATP concentration oscillations in proliferating cells}

In their study, Martin and Müller [53] hypothesized that the eukaryotic cells' common ancestor arose from a symbiotic process between an anaerobic, autotrophic and "strictly hydrogen-dependent archaebacterium" and a respiring eubacterium, releasing hydrogen "as a waste product of anaerobic heterotrophic metabolism". This symbiosis between the hydrogen-dependent host and the symbiont, which produce hydrogen, is an attractive hypothesis supporting P. Mitchell's chemiosmotic theory of oxidative phosphorylation-dependent ATP synthesis in mitochondria [49]. In this model, it is assumed that the proton gradient across the internal membrane drives the electron transport through the ETC. As reported above, this is performed by transmembrane 
complexes, which pump protons from the matrix to the intermembrane space. Then this gradient triggers ATP synthase activity and ATP synthesis.

Further, studies explain the energy of the cell from [ATP]/[ADP] ratio point of view. Understanding the bioenergetics of a normal dividing cell at the scale of the entire cell cycle may bring some interesting answers to the cancer cell phenotype [7]. Interestingly, independent studies reported the oscillation of the intracellular ATP concentration through the cell cycle [13] and its extensive use in ionic pump activity [54]. Marcussen and colleagues (1992) reported on ATP concentration oscillation along the progression of the cell cycle [13]. They found that the ATP concentration is minimal at the $G_{1} / S$ phase transition and then progressively reaches its maximum at $G_{2} /$ Mitosis (Fig. 2). This correlates with the extensive studies by Boonstra and colleagues who reported ATP hydrolysis-dependent $\mathrm{Na}^{+}-\mathrm{K}^{+}$ATPase pump activity during the $\mathrm{G}_{1} / \mathrm{S}$ transition cell cycle $[54,55]$. This pump is known to be responsible for the electric potential of large populations of cells by extruding three $\mathrm{Na}^{+}$and against the two $\mathrm{K}^{+}$influx. This is confirmed here, where Veech and colleagues [55] showed that intracellular ATP hydrolysis is tightly linked to $\mathrm{Na}^{+}-\mathrm{K}^{+} \mathrm{ATPase}$ pump activity, responsible for the inherent oscillation of the electrical potential of the cell [56] (Fig. 3). Consequently, ATP/ADP ratio oscillation modulated by ATP synthesis in mitochondria and hydrolysis in the entire cytoplasm, throughout the cell cycle, is also linked to another intracellular oscillator, i.e., intracellular $\mathrm{pH}(\mathrm{pHi})$.

\section{Intracellular $\mathrm{pH}$ oscillates in phase with $A T P / A D P$ ratio}

Increasing evidences indicate that the intracellular $\mathrm{pH}(\mathrm{pHi})$ homeostasis is correlated with cell metabolism and proliferation [57-60]. Aerts and colleagues (1985) experimentally showed an autonomous pHi cycle within a Dictyostelium cell. They managed to demonstrate that modulating $\mathrm{pHi}$ controls protein synthesis and DNA replication: optimal protein and DNA synthesis being correlated with an alkaline pHi of 7.4. This is confirmed by other studies showing the pHi-dependent enzyme activity [61-63]. In their studies, Busa et al. [62] showed that pHi oscillations are master regulators in the decision-making of brine shrimp embryonic cells to enter dormancy or to continue development: the acidic $\mathrm{pHi}$ is linked to dormancy, whereas the alkaline pHi is characteristic of development. In 1983, Christen et al. highlighted the intertwined relationship between intracellular $\mathrm{pH}$, ATP hydrolysis, and mitochondrial respiration [38]. At acidic $\mathrm{pH}$, the cytoplasmic activity of dynein ATPase, which is involved in microtubule dynamics, is inhibited and the internal ATP concentration is high, translating optimal mitochondrial respiration. On the other hand, when pHi is alkaline, the cytoplasmic ATP concentration falls, probably due to impaired mitochondrial respiration and increased dynein ATPase activity [64]. The authors concluded on the cytoplasmic $\mathrm{pH}$ control of ATP hydrolysis, inhibited in acidic pHi and enhanced in alkaline $\mathrm{pHi}$, and mitochondrial respiration increased triggered in acidic $\mathrm{pHi}$ and inhibited along with an increased alkaline $\mathrm{pHi}$.

\section{Intracellular $\mathrm{pH}$ times cell cycle entry and cell growth}

The intracellular $\mathrm{pH}$ change can be explained by several phenomena [65]. The sodium/ hydrogen exchanger, $\mathrm{Na}^{+}-\mathrm{H}^{+}-1$ (NHE-1), plays a significant role, especially in pHi alkalinization [66] (Fig. 3). Moolenaar and colleagues (1981) set up a series of experiments showing the role of NHE-1 in regulating the pHi. Addition of sodium to a 
neuroblastoma cell culture medium is followed by $\mathrm{Na}^{+}$uptake and $\mathrm{H}^{+}$extrusion. In 2000, Reshkin et al. did transfect normal cells by Human Papillomavirus (HPV), and observed that over-expression of NHE-1 is accompanied by cytoplasmic alkalinity which is first event of carcinogenesis. Recent studies confirm the crucial role of NHE-1 in preventing further cytoplasmic acidification [67-71]. More specifically, NHE-1 activity has been shown to be a key regulator of eukaryotic cell cycle entry and cell growth [69, 72, 73]. Moreover, Pouysségur and colleagues (1985) showed that under growth factor stimulation, the NHE-1 antiporter elevates the cytoplasmic $\mathrm{pH}$ of quiescent fibroblasts above a threshold of 7.2, a necessary step for cell cycle entry and DNA synthesis in $S$ phase [63]. Interestingly, some other studies showed a link between the metabolic state of the proliferating cells and histone acetylation [73, 74] (Fig. 4).

Indeed, Histone acetylation plays a pivotal role in regulating gene accessibility to RNA polymerase, for gene transcription [75]. Specific families of enzymes, called Histone Acetyltransferases (HATs) and Histone Deacetylases (HDACs), are responsible for histone acetylation and histone deacetylation, respectively. In this study [73], the authors reported the pivotal role of the $\mathrm{NAD}^{+} / \mathrm{NADH}$ ratio on sirtuin (HDACs) activity. The dynamics of histone acetylation has also been shown to be closely linked to pHi [76, 77]. In this study [76], McBrian and colleagues, showed that histone acetylation has the power of regulating the $\mathrm{pHi}$. The acidic $\mathrm{pHi}$ is indeed followed by global histone deacetylation and, thus global histone compaction. This is typically the phenotype of a dormant cell such as the one described above. Conversely, the pHi increase towards alkalinization is reported to favor global acetylation of histone, similarly to when "resting cells are induced to proliferate". Altogether, these results suggest the intertwined relationship between the metabolic cell cycle balancing the $\mathrm{NAD}^{+} / \mathrm{NADH}$ and ATP/ADP ratios through intracellular $\mathrm{pH}$ oscillations.

\section{Conclusion}

Proliferating cells must double their biomass (proteins, lipids, and nucleic acids) through the cell cycle in order to generate two daughter cells. For that they use the central carbon metabolism (CCM), universally shared among living systems. The CCM is governed by pivotal metabolic pathways such as glycolysis, the pentose phosphate pathway, and the citric acid cycle. The cell decision-making to enter one of these pathways is coupled to redox transitions following nutrient availability. In the CCM, nutrients, such as glucose and glutamine, are used to generate precursors through redox reactions and to support cell growth. Moreover, experimental studies show that the mitochondrial activity is reduced during early progression in the cell cycle in $G_{1}$ [32]. Also, the $G_{1}$ phase of the cell cycle is characterized by an anabolic demand in protein synthesis, required for DNA replication in $\mathrm{S}$ phase. Synthesis of building blocks, such as amino acids and DNA or pyruvate from carbohydrate pathways is then a necessary step for biomass synthesis and energy supply through mitochondrial activity.

Moreover, one understands that cells decision-making to enter proliferation or stay in dormancy depends on physical, electrical and biochemical parameters. Nutrients and growth hormones availability in the extracellular medium modulates all these three parameters since they provoke osmotic pressure resulting in variation 


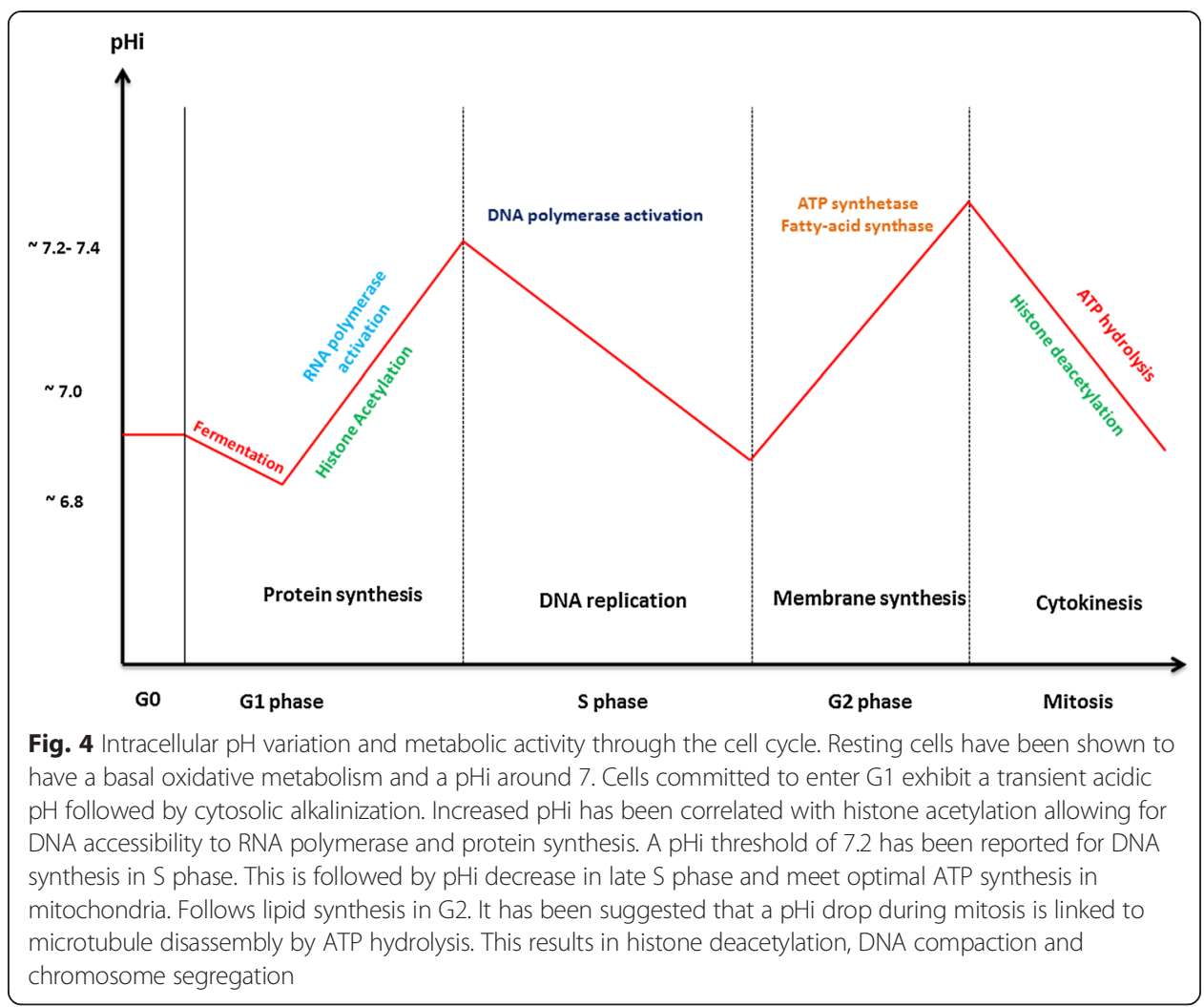

in bioelectrical parameters of the cell, such as the transmembrane potential, enzyme and cofactors charges and the intracellular (pHi). Perturbing one of these parameters has been reported to change the others. So that, cell metabolism seems to be the result of intertwined state parameter oscillations. In this literature investigation, we deciphered cell cycle progression from cell metabolism or more precisely central carbon metabolism (CCM) point of view. It appeared, first, the intriguing relationship between $\mathrm{CCM}$ and cell cycle progression, with the reactive and oxidative (redox) cofactors such as $\mathrm{NAD}^{+} / \mathrm{NADH}, \mathrm{NADP}^{+} / \mathrm{NADPH}$ being key regulators. Secondly, as reported, mitochondria seem to be more than just a plant for ATP synthesis. They are at the core of eukaryotic cell metabolism and cell cycle progression. In there, the tricarboxylic acid (TCA) cycle, branched to glycolysis and to the pentose phosphate pathway, is central in mitochondrial metabolism and has been reported to match mitosis. The TCA is also an adaptive circuit at the crossroads between cytosolic-mitochondrial energy exchanges which are especially enhanced when resting cells are committed to divide. Finally, the progression of the cell cycle exhibits a shifted metabolism, materialized by a shunt from catabolism to anabolism. Transitions are performed by redox potential variation, involving $\mathrm{NAD}^{+} / \mathrm{NADH}, \mathrm{NADP}^{+} / \mathrm{NADPH}$ redox couples, and ADP/ATP energetic ratios and the intracellular $\mathrm{pH}$ seems to the be master operator of cytosol/ mitochondrial flux balances. Understanding the dynamics of these metabolic exchanges will pave the way to therapeutic solutions for metabolic cycle disorders such as cancer. 


\section{Abbreviations}

DNA: Deoxyribonucleic acid; RNA: Ribonucleic acid; NAD: Nicotinamide adenine dinucleotide; NADP: Nicotinamide adenine dinucleotide phosphate; ATP: Adenosine triphosphate; ADP: Adenosine diphosphate; CCM: Central carbon metabolism; TCA: Tricarboxylic acid; PPP: Pentose phosphate pathway; $\mathrm{CO}_{2}$ : Carbon dioxide; ETC: Electron transport chain; G3P: Glyceraldehyde 3-phosphate; ROS: Reactive oxygen species; F6P: Fructose 6-phosphate; G6PDH: Glucose-6-phosphate dehydrogenase; GSH: Glutathione; GSSH: Mitochondrial pyruvate carrier; FAD: Flavin adenine dinucleotide; NHE-1: Sodium-hydrogen exchanger 1; NaK-ATPase: Sodium-potassium ATPase; HATs: Histone acetyltransferases; HDACs: Histone deacetylase.

\section{Competing interests}

The authors declare that they have no competing interests.

\section{Authors' contributions}

LS, JMS and JDM deciphered the key points developed in this review and wrote the manuscript. SP also participated in the description of the central carbon metabolism and designed the relating figures. EB, LP and ML participated in discussing CCM transitions and provided edition and critical review. All authors read and approved the final manuscript.

\section{Acknowledgements}

We would like to especially thank Khalid Omer Alfarouk for revising this article and for his suggestions. Johannes Boonstra is also gratefully acknowledged for sending us his "not-easy-to-find" articles. Last but not least, we deeply thank "Association Cancer \& Métabolisme" and Bertrand Halff.

\section{Author details}

${ }^{1}$ Ecole Polytechnique, LIX-UMR 7161, Palaiseau, France. ' 2 RI, Paris-Sud University, CNRS UMR8623 and INRIA Saclay, Paris, France. ${ }^{3}$ Paris Institute of Translationnal Neurosciences (IHU-A-ICM), Pitié Salpêtrière Hospital, Paris, France.

Received: 18 February 2015 Accepted: 15 May 2015

Published online: 29 May 2015

\section{References}

1. Lane N, Martin WF. The origin of membrane bioenergetics. Cell. 2012;151(7):1406-16.

2. Tyson JJ. Biochemical oscillations. In: Computer cell Biology. New York: Springer-Verlag; 2002. p. 230-60.

3. DeBerardinis RJ, Lum JJ, Hatzivassiliou G, Thompson CB. The biology of cancer: metabolic reprogramming fuels cell growth and proliferation. Cell Metab. 2008;7(1):11-20.

4. Alfarouk KO, Verduzco D, Rauch C, Muddathir AK, Adil HH, Elhassan GO, et al. Glycolysis, tumor metabolism, cancer growth and dissemination. A new pH-based etiopathogenic perspective and therapeutic approach to an old cancer question. Oncoscience. 2014:1(12):777-802.

5. Uzman A. Molecular Cell Biology, Sixth Edition. Biochem Mol Biol Educ. 2010;38:60-1.

6. Fabregat I, Revilla E, Machado A. The NADPH consumption regulates the NADPH-producing pathways (pentose phosphate cycle and malic enzyme) in rat adipocytes. Mol Cell Biochem. 1987;81:77-81.

7. Diaz-Moralli S, Tarrado-Castellarnau M, Miranda A, Cascante M. Targeting cell cycle regulation in cancer therapy. Pharmacol Ther. 2013;138(2):255-71.

8. Noor E, Eden E, Milo R, Alon U. Central carbon metabolism as a minimal biochemical walk between precursors for biomass and energy. Mol Cell. 2010;39(5):809-20.

9. Bar-Even A, Flamholz A, Noor E, Milo R. Rethinking glycolysis: on the biochemical logic of metabolic pathways. Nat Chem Biol. 2012;8(6):509-17.

10. Fell DA. Evolution of central carbon metabolism. Mol Cell. 2010;39(5):663-4.

11. Calderon-Montano J, Burgos-Moron E, Perez-Guerrero C, Salvador J, Robles A, Lopez-Lazaro M. Role of the Intracellular $\mathrm{pH}$ in the Metabolic Switch between Oxidative Phosphorylation and Aerobic Glycolysis - Relevance to Cancer. WebmedCentral CANCER. 2011;2(3):WMC001716.

12. Harguindey S, Arranz JL, Polo Orozco JD, Rauch C, Fais S, Cardone RA, et al. Cariporide and other new and powerful NHE1 inhibitors as potentially selective anticancer drugs-an integral molecular/biochemical/metabolic/ clinical approach after one hundred years of cancer research. J Transl Med. 2013;11:282.

13. Marcussen M, Larsen PJ, Anatomy M, Panum T. Cell Cycle-Dependent Regulation of Cellular ATP Concentration, and Depolymerization of the Interphase Microtubular Network Induced by Elevated Cellular ATP Concentration in Whole Fibroblasts. Cell Motil Cytoskeleton. 1996;35(2):94-9.

14. Yu FX, Dai RP, Goh SR, Zheng L, Luo Y. Logic of a mammalian metabolic cycle: An oscillated NAD+/NADH redox signaling regulates coordinated histone expression and S-phase progression. Cell Cycle. 2009;8:773-9.

15. Warburg O. On the origin of cancer cells. Science. 1956;123(3191):309-14.

16. Demetrius LA, Coy J, Tuszynski JA. Cancer proliferation and therapy: the Warburg effect and quantum metabolism. Theor Biol Med Model. 2010;2-7.

17. Davies PC, DemetriusL LA, Tuszynski JA. Cancer as a dynamical phase transition. Theor Biol Med Model. 2011;8-30.

18. Rietman EA, Friesen DE, Hahnfeldt P, Gatenby R, Hlatky L, Tuszynski JA. An integrated multidisciplinary model describing initiation of cancer and the Warburg hypothesis. Theor Biol Med Model. 2013;10:39.

19. Burhans CW, Heintz NH. The cell cycle is a redox cycle: linking phase-specific targets to cell fate. Free Radic Biol Med. 2009:47(9):1282-93.

20. Chiu J, Dawes IW. Redox control of cell proliferation. Trends Cell Biol. 2012;22(11):592-601.

21. Menon SG, Goswami PC. A redox cycle within the cell cycle: ring in the old with the new. Oncogene. 2007;26(8):1101-9. 
22. Sarsour E, Kumar M. Redox control of the cell cycle in health and disease. Antioxid Redox Signal. 2009;11(12):2985-3011.

23. Jenuwein T, Allis CD. Translating the histone code. Science. 2001;293(5532):1074-80.

24. Morinobu A, Kanno Y, O'Shea JJ. Discrete roles for histone acetylation in human T helper 1 cell-specific gene expression. J Biol Chem. 2004;279(39):40640-6.

25. Choudhury M, Park PH, Jackson D, Shukla SD. Evidence for the role of oxidative stress in the acetylation of histone H3 by ethanol in rat hepatocytes. Alcohol. 2010;44:531-40.

26. Smith CL. A shifting paradigm: histone deacetylases and transcriptional activation. Bioessays. 2008;30(1):15-24.

27. Fabregat I, Vitorica J, Satrustegui J, Machado A. The pentose phosphate cycle is regulated by NADPH/NADP ratio in rat liver. Arch Biochem Biophys. 1985;236:110-8.

28. Fabregat I, Revilla E, Machado A. Short-term control of the pentose phosphate cycle by insulin could be modulated by the NADPH/NADP ratio in rat adipocytes and hepatocytes. Biochem Biophys Res Commun. 1987;146:920-5.

29. Revilla E, Fabregat I, Santa María C, Machado A. The NADPH-producing pathways (pentose phosphate and malic enzyme) are regulated by the NADPH consumption in rat mammary gland. Biochem Int. 1987;14:957-62.

30. Vanamala I, Radhakrishnan S, Reddivari L, Bhat VB, Ptitsyn A. Resveratrol suppresses human colon cancer cel proliferation and induces apoptosis via targeting the pentose phosphate and the talin-FAK signaling pathways-A proteomic approach. Proteome Sci. 2011;9(1):49.

31. Eggleston LV, Krebs HA. Regulation of the pentose phosphate cycle. Biochem J. 1974;138:425-35.

32. Monod J, Wyman J, Changeux JP. On the Nature of Allosteric Transitions: a Plausible Model. J Mol Biol. $1965 ; 12: 88-118$.

33. Han CY, Umemoto T, Omer M, Den Hartigh L, Chiba T, LeBoeuf R, et al. NADPH oxidase-derived reactive oxygen species increases expression of monocyte chemotactic factor genes in cultured adipocytes. J Biol Chem. 2012;287:10379-93

34. Winkler BS, DeSantis N, Solomon F. Multiple NADPH-producing pathways control glutathione (GSH) content in retina. Exp Eye Res. 1986;43:829-47.

35. McBride H, Neuspiel M, Wasiak S. Mitochondria: more than just a powerhouse. Curr Biol. 2006:16(14):R551-6.

36. Hackenbrock CR. Ultrastructural bases for metabolically linked mechanical activity in mitochondria. I. Reversible ultrastructural changes with change in metabolic steady state in isolated liver mitochondria. J Cell Biol. 1966;30:269-97.

37. Scalettar BA, Abney RJ, Hackenbrock CR. Dynamics, structure, and function are coupled in the mitochondrial matrix. Proc Natl Acad Sci U S A. 1991;88:8057-61.

38. Christen R, Schackmannfl RW, Shapiroll M. Metabolism of sea urchin sperm. Interrelationships between intracellular pH, ATPase activity, and mitochondrial respiration. J Biol Chem. 1983;258(9):5392-9.

39. Jones RG, Plas DR, Kubek S, Buzzai M, Mu J, Xu Y, et al. AMP-activated protein kinase induces a p53-dependent metabolic checkpoint. Mol Cell. 2005;18:283-93.

40. Hardie DG. New roles for the LKB1 $\rightarrow$ AMPK pathway. Curr Opin Cell Biol. 2005;17:167-73.

41. Mandal S, Guptan P, Owusu-Ansah E, Banerjee U. Mitochondrial regulation of cell cycle progression during development as revealed by the tenured mutation in Drosophila. Dev Cell. 2005;9(6):843-54.

42. Mitra K, Wunder C, Roysam B, Lin G, Lippincott-Schwartz J. A hyperfused mitochondrial state achieved at G1-S regulates cyclin E buildup and entry into S phase. Proc Natl Acad Sci U S A. 2009:106(29):11960-5

43. Herzig S, Raemy E, Montessuit S, Veuthey JL, Zamboni N, Westermann B, et al. Identification and Functional Expression of the Mitochondrial Pyruvate Carrier. Science. 2012;337:93-6.

44. Bricker DK, Taylor EB, Schell JC, Orsak T, Boutron A, Chen YC, et al. A Mitochondrial Pyruvate Carrier Required for Pyruvate Uptake in Yeast, Drosophila, and Humans. Science. 2012;337:96-100.

45. Halestrap AP. The mitochondrial pyruvate carrier: Has it been unearthed at last. Cell Metab. 2012;16:141-3.

46. Beaulieu C. The basis of anisotropic water diffusion in the nervous system-A technical review. NMR Biomed. 2002;15:435-55.

47. Fendt SM, Bell EL, Keibler MA, Olenchock BA, Mayers JR, Wasylenko TM, et al. Reductive glutamine metabolism is a function of the a-ketoglutarate to citrate ratio in cells. Nat Commun. 2013;4:2236.

48. Israël M. Signaling And Metabolism In Cancer: Endocrine Pancreas Deficiency And Hybrid Anabolism-Catabolism. Drugs That Undo The Process. Cancer Ther. 2014;10:1-12.

49. Krauss S. Mitochondria: Structure and role in respiration. Nature Publishing Group, Encyclopedia of Life Sciences (2001) p. 6

50. Mitchell P. Coupling of phosphorylation to electron and hydrogen transfer by a chemi-osmotic type of mechanism. Nature. 1961;191:144-8.

51. Finley LWS, Zhang J, Ye J, Ward PS, Thompson CB. SnapShot: cancer metabolism pathways. Cell Metab. 2013;17(3):466-6. e2.

52. Gagliardi LJ, Shain DH. Is intracellular pH a clock for mitosis. Theor Biol Med Model. 2013;10(1):8.

53. Martin W, Müller A. The hydrogen hypothesis for the first eukaryote. Nature. 1998;392(6671):37-41.

54. Mummery CL, Boonstra J, Van Der Saag PT, de Laat SW. Modulation of functional and optimal (Na + -K+)ATPase activity during the cell cycle of neuroblastoma cells. J Cell Physiol. 1981;107:1-9.

55. van Zoelen EJ, Mummery CL, Boonstra J, van der Saag PT, de Laat SW. Membrane regulation of the Na+, K+-ATPase during the neuroblastoma cell cycle: correlation with protein lateral mobility. J Cell Biochem. 1983;21:77-91.

56. Veech RL, Kashiwaya Y, Gates DN, King MT, Clarke K. The Energetics of Ion Distribution : The Origin of the Resting Electric Potential of Cells. IUBMB Life. 2002;54(5):241-52.

57. Aerts RJ, Durston AJ, Moolenaar WH. Cytoplasmic pH and the regulation of the Dictyostelium cell cycle. Cell. 1985:43(3 Pt 2):653-7.

58. Busa W, Nuccitelli R. Metabolic regulation via intracellular pH,". Am J Physiol. 1984;246(4 Pt 2):R409-38.

59. Cohen A, Doveh E, Eick U. Statistical properties of the $r(W G(J))$ index of agreement. Psychological Methods. $2001 ; 6: 297-310$ 
60. Ciapa B, Philippe L: Intracellular and Extracellular pH and Ca Are Bound to Control Mitosis in the Early Sea Urchin Embryo via ERK and MPF Activities. PLoS One, vol. 8, 2013.

61. Busa WB, Crowe JH, Matson GB. Intracellular pH and the metabolic status of dormant and developing Artemia embryos. Arch Biochem Biophys. 1982;216:711-8.

62. Busa WB, Crowe JH. Intracellular pH Regulates Transitions Between Dormancy and Development of Brine Shrimp (Artemia salina) Embryos. Science. 1983;221:366-8.

63. Hand SC, Carpenter JF. pH-Induced Metabolic Transitions in Artemia Embryos Mediated by a Novel Hysteretic Trehalase. Science. 1986;232:1535-7.

64. Summers KE, Gibbons IR. Adenosine Triphosphate-Induced Sliding of Tubules in Trypsin-Treated Flagella of Sea-Urchin Sperm. Proc Natl Acad Sci. 1971;68(12):3092-6.

65. Boron W. Intracellular pH regulation. Adv Physiol Educ. 2004;28(1-4):160-79.

66. Moolenaar WH, Boonstra J, van der Saag PT, de Laat SW. Sodium/proton exchange in mouse neuroblastoma cells. J Biol Chem. 1981;256(24):12883-7.

67. Sardet C, Franchi A, Pouysségur J. Molecular cloning, primary structure, and expression of the human growth factor-activatable $\mathrm{Na}+/ \mathrm{H}+$ antiporter. Cell. 1989:56(2):271-80.

68. Bianchini L, Pousségur J. Molecular structure and regulation of vertebrate $\mathrm{Na}+/ \mathrm{H}+$ exchangers. J Exp Biol. 1994;196:337-45

69. Paris S, Pouyssegur J. Growth Factors Activate the $\mathrm{Na}+/ \mathrm{H}+$ Antiporter in Quiescent Fibroblasts by Increasing Its Affinity for Intracellular H+. J Biol Chem. 1984;259(17):10989-94.

70. Lacroix J, Poët M, Maehrel C, Counillon L. A mechanism for the activation of the $\mathrm{Na} / \mathrm{H}$ exchanger NHE-1 by cytoplasmic acidification and mitogens. EMBO Rep. 2004;5(1):91-6.

71. Counillon L, Pouysségur J. The expanding family of eucaryotic Na+/H+ exchangers. J Biol Chem. 2000;3(33):1-4.

72. Pouysségur J, Franchi A, L'Allemain G, Paris S. Cytoplasmic $\mathrm{pH}$, a key determinant of growth factor-induced DNA synthesis in quiescent fibroblasts. FEBS Lett. 1985;190(1):115-9.

73. Wellen KE, Hatzivassiliou G, Sachdeva UM, Bui TV, Cross JR, Thompson CB. ATP-citrate lyase links cellular metabolism to histone acetylation. Science. 2009;324(5930):1076-80.

74. Vogelauer M, Krall AS, McBrian MA, Li JY, Kurdistani SK. Stimulation of histone deacetylase activity by metabolites of intermediary metabolism. J Biol Chem. 2012;287(38):32006-16.

75. Kurdistani SK, Grunstein M. Histone acetylation and deacetylation in yeast. Nat Rev Mol Cell Biol. 2003;4:276-84.

76. McBrian MA, Behbahan IS, Ferrari R, Su T, Huang TW, Li K, et al. Histone acetylation regulates intracellular pH. Mol Cell. 2013:49(2):310-21.

77. Kurdistani SK. Chromatin : a capacitor of acetate for integrated regulation of gene expression and cell physiology. Curr Opin Genet Dev. 2014;26(Figure 1):53-8.

\section{Submit your next manuscript to BioMed Central and take full advantage of:}

- Convenient online submission

- Thorough peer review

- No space constraints or color figure charges

- Immediate publication on acceptance

- Inclusion in PubMed, CAS, Scopus and Google Scholar

- Research which is freely available for redistribution

Submit your manuscript at www.biomedcentral.com/submit 